\title{
Acknowledgement to Reviewers of Urban Science in 2019
}

\author{
Urban Science Editorial Office
}

MDPI AG, St. Alban-Anlage 66, 4052 Basel, Switzerland

Published: 28 January 2020

The editorial team greatly appreciates the reviewers who have dedicated their considerable time and expertise to the journal's rigorous editorial process over the past 12 months, regardless of whether the papers are finally published or not. In 2019, a total of 110 papers were published in the journal, with a median time to first decision of 13.2 days and a median time from submission to publication of 42 days. The editors would like to express their sincere gratitude to the following reviewers for their generous contribution in 2019:

$\begin{array}{ll}\text { Abarca-Alvarez, Francisco Javier } & \text { Li, Zhenlong } \\ \text { Adam, Paul } & \text { Li, Chao-Su } \\ \text { Addie, Jean-Paul } & \text { Li, Tao } \\ \text { Afonso, Armando Silva } & \text { Liang, Donghai } \\ \text { Ainiala, Terhi } & \text { Licitra, Gaetano } \\ \text { Alonso, Alicia } & \text { Lindholm, Gunilla } \\ \text { Alsina-Pagès, Rosa Ma } & \text { Lipp, Torsten } \\ \text { Alves, Fernando Brandão } & \text { Liu, Zhengchun } \\ \text { Amirkhani, Mehdi } & \text { Liu, Ye } \\ \text { Anderson, Elsa } & \text { Liu, Jiechao } \\ \text { Anderson, Paul } & \text { Loppi, Stefano } \\ \text { Annis, Antonio } & \text { Love, Steve } \\ \text { Anz, Craig } & \text { Luis Ubago, Jose } \\ \text { Araldi, Alessandro } & \text { MacKenzie, Andrew } \\ \text { Aranburu, Ibon } & \text { Mansour, Osama E. } \\ \text { Araújo, Noelia } & \text { Mao, Yong } \\ \text { Arias Trujillo, Juana } & \text { Maretto, Marco } \\ \text { Armstrong, Helen } & \text { Mazzulla, Gabriella } \\ \text { Arroyo, Rosa } & \text { McClure, Kirk } \\ \text { Atazadeh, Behnam } & \text { Meenar, Mahbubur } \\ \text { Attwater, Roger } & \text { Mehaffy, Michael } \\ \text { Azevedo Guedes, André Luis } & \text { Mendoza, Daniel } \\ \text { Bai, Yun } & \text { Miao, Julie Tian } \\ \text { Balsa-Barreiro, Jose } & \text { Mirlohi, Susan } \\ \text { Bartkowski, John } & \text { Moccia, Francesco Domenico } \\ \text { Bathrellos, George } & \text { Montealegre, María Grinan } \\ \text { Battarra, Rosaria } & \text { Morales-Alonso, Gustavo } \\ \text { Beckmann, Volker } & \text { Moreno, Ana Tsui } \\ \text { Benassi, Federico } & \text { Morgan, Edward } \\ \text { Betancur, John J } & \text { Mortara, Michela } \\ \text { Bieda, Agnieszka } & \text { Müller, Bernhard } \\ \text { Bigon, Liora } & \text { Mundula, Luigi } \\ \text { Boanca, Paunita } & \\ \text { Aurgante, Beniamino } \\ \text { And }\end{array}$


Boeing, Geoff

Bolton, Natalie

Borghi, Guido

Bourassa, Steven C.

Bouzguenda, Islam

Brimblecombe, Peter

Brinia, Vasiliki

Bronisz, Urszula

Burgalassi, David

Cabral-Pinto, Marina M. S.

Caldarice, Ombretta

Calzada, Igor

Cambra, Paulo

Campagnaro, Thomas

Campisi, Tiziana

Camporeale, Rosalia

Cao, Kai

Carles Arribas, José Luis

Cartalis, Constantinos

Castanho, Rui Alexandre

Cebrián-Abellán, Francisco

Celani, Alberto

Chambel, Luis

Chen, Zifeng

Chen, Xueming (Jimmy)

Chenal, Jérôme

Choo, Pei Ling

Clark, Thomas A

Coccossis, Harry

Corazza, Maria Vittoria

Costa, Nuno Manuel Sessarego Marques Da

Cruz, Carlos Oliveira

D'Autilia, Roberto

D'Acci, Luca

Daldanise, Gaia

Dallari, Fiorella

Dane, Gamze Z.

David, João

de la Vega, Alfonso Garcia

Deilami, Kaveh

Delmelle, Elizabeth

Delotto, Roberto

Dettori, Marco

DeVereaux, Constance

Diaz-Sarachaga, Jose Manuel

Doevendans, Kees

Douglass, Matthew

Drago, Carlo

$\mathrm{Du}$, Xiaomin

Đurkin Badurina, Jelena

Earley-Spadoni, Tiffany

Elsen, Suanne

Emo, Beatrix
Mydlarz, Charlie

Myeong, Seunghwan

Nalmpantis, Dimitrios

Natarajan, Lucy

Naumov, Nikola

Nikitas, Alexandros

Nofal, Eslam

Nucci, Lucia

Nunn, Patrick

Obeng-Gyasi, Emmanuel

Oberman, Tin

Opdyke, Aaron

Oust, Are

Pafka, Elek

Palazzo, Anna Laura

Palella, Boris Igor

Pan, Haozhi

Panda, Bibhudutta

Paolicelli, Federica

Papageorgiou, Marilena

Paraskevopoulou, Angeliki

Park, Moo Jong

Park, Sojung

Pérez Sánchez, Modesto

Pérez-Campaña, Rocío

Pettit, Chris

Philippopoulos, Kostas

Piccioni, Cristiana

Piguet, Etienne

Piñeira-Mantiñán, María José

Pino, Joaquín Martínez

Pitidis, Vangelis

Poelling, Bernd

Pont, Ulrich

Potrafke, Niklas

Pritoni, Marco

Privitera, Donatella

Quaquero, Emanuela

Quinn, Casey

Ramos-Vidal, Ignacio

Ratcliffe, Eleanor

Reece, Jason

Ribeiro, Ana Isabel

Ribeiro, Jorge Tavares

Rodrigues, Evelina

Rong, Li

Rossetti, Silvia

Rotondo, Francesco

Russo, Alessio

Salingaros, Nikos

Sanchez, Thomas

Santini, Erica

Sargolini, Massimo 
Encarnação, Sara

Erlandsson, Lan Wang

Fabbri, Kristian

Falcone, James A.

Fernández-Revelles, Andrés B.

Ferreiro, Maria Fátima

Fewkes, Alan

Flies, Emily

Foster, Alec

Fragoso, António

Fredianelli, Luca

Frizziero, Leonardo

Futcher, Julie

Gabriel, Richard

Gál, Tamás

Gallo, Mariano

Garnero, Gabriele

Gearey, Mary

Genc, Elif

Georgiadis, Teodoro

Gharebaghi, Amin

Giuffrida, Nadia

Gorgitano, Maria Teresa

Grafius, Darren

Granberry, Phillip J.

Green, Andrew

Growe, Anna

Hamiduddin, Iqbal

Harkin, Kenneth R.

Hashemi-Beni, Leila

Heiland, Stefan

Hernández-Palacio, Fabio

Hertig, Elke

Holvad, Torben

Homsy, George

Howell, Aaron

Huynh, Neil

Ioakimidis, Christos

Jang, Sunghoon

Jauhiainen, Jussi

Jeannotte, Sharon

Jimenez, Emilio

Jonas, Andrew

Jun, Sunghae

Kang, Jeon-Young

Kapetas, Leon

Karvonen, Andrew

Keler, Andreas

Keough, Sara Beth

Kim, Jae Ik

Kim, Ki Pyung

Kim, Inhi

Kim, Karl

King, David A.
Schaefer, Klaus

Sciuto, Gaetano Antonio

Seamon, David

Sedrez, Maycon

Settembre Blundo, Davide

Setty, Karen

Shafique, Muhammad

Shahab, Sina

Shaheen, Susan

Shan, Xiaojun (Gene)

Shen, Yao

Shirvani Dastgerdi, Ahmadreza

Shokrolah Shirazi, Mohammad

Sikder, Sujit Kumar

Silva, Cristian

Simpson, Greg D.

Sivula, Anna

Sobral, Thiago

Soriano, Antonio

Souto, Ana

Stangl, Paul

Stavroulakis, Georgios E.

Stephenson, Max O.

Stewart, Rodney

Stewart, Alex G.

Stober, Dina

Stokes, Lynissa

$\mathrm{Su}, \mathrm{Ye}$

Susan, Buckingham

Swapan, Mohammad

Szczepańska, Agnieszka

Taylor, John R.

Tendero, Marjorie

Tepe, Emre

Tregua, Marco

Trivic, Zdravko

Trovato, Maria Rosa

Tselios, Vassilis

Tsilimigkas, Georgios

Tsitsifli, Stavroula

$\mathrm{Tu}$, Min-Cheng

Ulbrich, Philipp

Uribe-Toril, Juan

Usui, Hiroyuki

Valcárcel-Aguiar, Beatriz

Valdmanis, Vivian

Van Hoof, Joost

Van Wee, Bert

Vaz, Jorge Vieira

Venco, Elisabetta Maria

Versaci, Antonella

Vicino, Thomas

Visvizi, Anna

Vranayová, Zuzana 
Klyn, Dan

Koohsari, Javad

Kotter, Richard

Kotval-Karamchandani, Zeenat

Kotzen, Benz

Koutamanis, Alexander

Kraniotis, Dimitrios

Kucheva, Yana

Kumar, Amit

Kuo, Hsing-Fu

Kyvelou, Stella Sofia

La Licata, Ivana

La Rocca, Rosa Anna

La Russa, Mauro

LABIANCA, Marilena

Lam, Bhan

Lamb, David S.

Laylavi, Farhad

Ledda, Antonio

Lee, Jae Min

Leonard, Rosemary Jill

Leung, Abraham

Lewi, Hannah

Lewis, Kathy
Wang, Jue

Wang, Yang

Watanabe, Toru

Whittinghill, Leigh

Wohlrabe, Klaus

Wu, Chih-Da

Xia, Jianhong (Cecilia)

Xiao, Jieling

Xie, Junfei

Yerena, Anaid

Yiannakou, Athena

$\mathrm{Yu}$, Zhen

Zaman, Atiq

Zambon, Ilaria

Zamora, Willian

Zanon, Bruno

Zeedan, Rami

Zeferino, João A.

Zhang, Charlie

Zhang, Qi

Zhao, Bin

Zivkovic, Ljiljana

Zoppi, Corrado

Zuniga-Teran, Adriana

(C) 2020 by the author. Licensee MDPI, Basel, Switzerland. This article is an open access article distributed under the terms and conditions of the Creative Commons Attribution (CC BY) license (http://creativecommons.org/licenses/by/4.0/). 